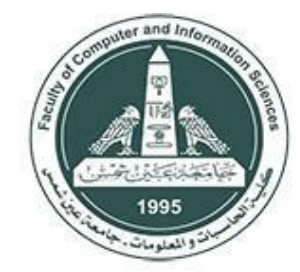

International Journal of Intelligent Computing and Information Sciences

https://ijicis.journals.ekb.eg/

\title{
APPLICATIONS OF COMPUTATIONAL INTELLIGENCE IN COMPUTER MUSIC COMPOSITION
}

\author{
Nermin Naguib J. Siphocly \\ Computer Science Department, \\ Faculty of Computer and \\ Information Sciences, Ain Shams \\ University \\ nermine.naguib@cis.asu.edu.eg \\ Received 2021-2-13; Revised 2021-3-7; Available online 2021-3-15
}

\author{
Abdel-Badeeh M. Salem \\ Computer Science Department, \\ Faculty of Computer and \\ Information Sciences, Ain Shams \\ University \\ absalem@cis.asu.edu.eg
}

\begin{abstract}
Engaging computers in composing musical pieces is a challenging and trending field of research. The musical tasks that can be performed or aided by computers' computational powers, are numerous. This paper is concerned with applications of computational intelligence in music composition. Its main objective is to survey various computational intelligence techniques for performing miscellaneous music composition tasks. To achieve this objective, we first define each music composition task, then we discuss the recent applications of each, and the techniques adopted in them. We also highlight the most suitable techniques for performing each task. Our study shows that the most suitable techniques for human composers imitative systems are case-based reasoning and artificial neural networks. It is also shown that Markov models are more suitable for predicting musical notes based on the given previous notes. Genetic algorithms excel in chord progressions generation. Deep neural networks are clever at capturing temporal information of a musical piece. The state-of-the-art generative adversarial networks produce music as close as possible to real compositions. At the end of this study, we shed the light on many future research directions in the field of computer music composition.
\end{abstract}

Keywords: Computer Music, Computational Intelligence, Artificial Intelligence, Algorithmic Composition

\section{Introduction}

Turning computers into music composers or even into aider for music composers, is very challenging. Composing musical pieces through computers requires, not only feeding computers with musical rules, but also teaching them to infer musical rules from already existing musical pieces. Thus, computer music generation is specifically challenging to the field of Computational Intelligence (CI) including also Artificial Intelligence (AI) and Machine Learning (ML) [1].

* Corresponding author: Nermin Naguib J. Siphocly

Computer Science Department, Faculty of Computer and Information Sciences, Ain Shams University

E-mail address: nermine.naguib@cis.asu.edu.eg 
In the last decade there has been a boost in the applications that involve computational intelligence techniques and algorithms. Computational intelligence techniques have been heavily involved in numerous applications related to various research fields such as face recognition through deep learning [2], Image retrieval through knowledge-based techniques [3]. Additionally, there are applications of AI in the field of medical research [4] and even in Covid-19 related research [5].

Computer music composition is a rather novel field of research. In this paper we aim for studying the modern applications of $\mathrm{CI}$ in the field of computer music composition. Music composition process is divided into several music composition tasks such as melody, accompaniment, and rhythm generation, each of which have further subtypes that will be discussed later in this paper. Additionally, CI, AI and ML have been excessively used in music composition field due to being able to simulate human creativity in a way or another. Figure. 1 includes the most popular intelligence algorithms (to the left) used in automating music composition tasks (to the right).

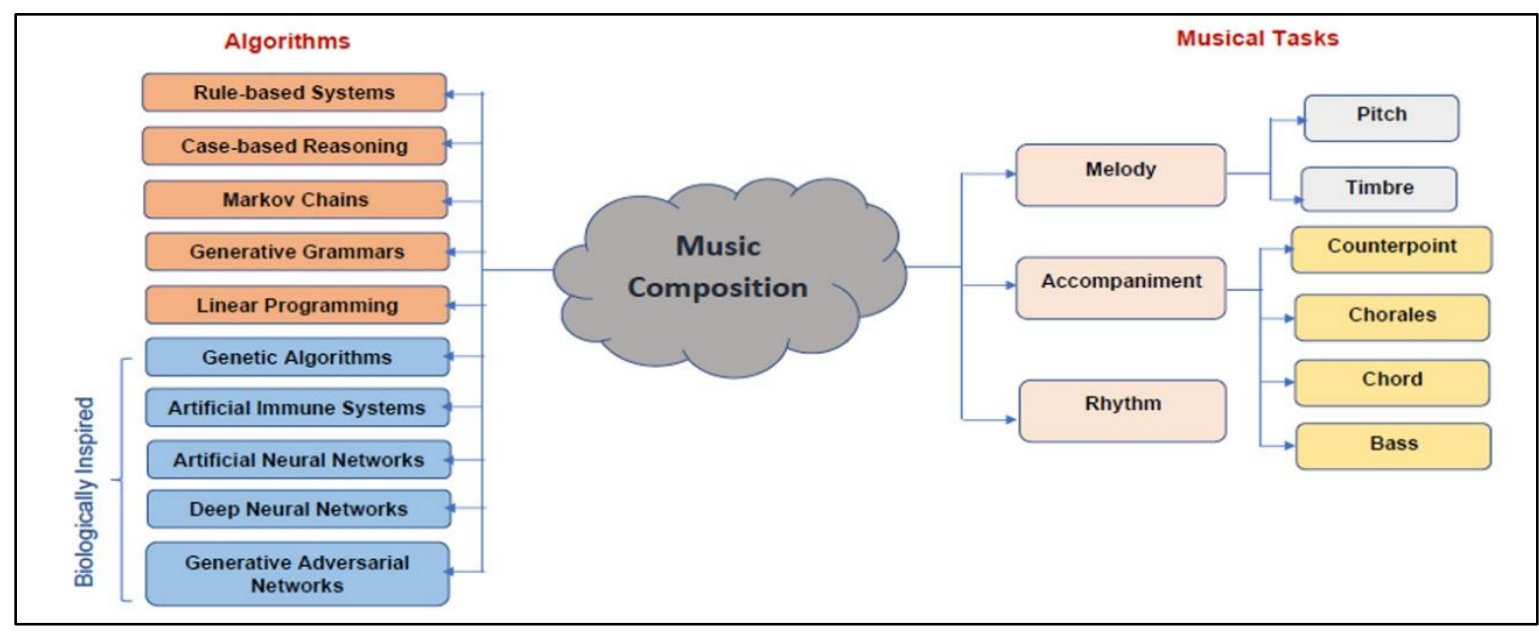

Figure. 1: Music Composition Tasks and Algorithms

In this paper we aim to give insight on which computational techniques are most suitable for which music composition tasks. We list the most recent applications adopting each technique. Finally, we discuss the future research directions related to them.

The rest of this paper is organized as follows Section 2 discusses the music composition systems classified by music compositions tasks. For each task, we illustrate the most recent applications and computational intelligence techniques adopted in them. Section 3 is a discussion of the findings of our study. Finally, we conclude our survey in Section 4 proposing some future research directions.

\section{Study of Music Composition Systems}

Computer music composition became highly correlated with the term "algorithmic composition" which, from its name, uses algorithms in automating the music composition process. The following subsections discuss recent research in automating each composition task.

\subsection{Melody Pitch}

A musical piece is mostly represented by its main melody. The melody is a set of single consecutive, satisfying, notes forming the musical piece. Musical notes' pitch distinguishes them from each other. A pitch is the human interpretation to sound wave frequency and is closely related to it. A primary objective to many music composition applications is to imitate a human composer's experience; thus, 
Case-Based Reasoning (CBR) makes a suitable approach for the task. María Navarro-Cáceres et al. [6] combined case-based reasoning with a Markov model in a melody composition system. Their objective was to obtain the probabilities of a given note following the last note incorporated in the melody.

Pedro et al. [7] proposed a multi-objective fitness function genetic algorithm (GA) for enhancing the selection process of melody generation. Moreover, they proposed "Melodic Trees" as a data structure for chromosomes representation.

Deep Neural Networks (DNNs) have a good share in music composition applications. Colombo et al. [8] developed a system for melody generation through deep Recurrent Neural Networks (RNNs) that is able to capture long-range temporal structure. Since Conditional Restricted Boltzmann Machine (CRBM) has the ability to model temporal dependencies, Benjamin Smith took advantage of this to achieve full reconstructions of musical pieces given a few starting seed note. From the very first DNNs to deal with audio data is WaveNet [9]. WaveNet was primarily developed for speech synthesis purposes; however, when experimented on musical piano pieces it was able to produce realistic musical waveforms. The first version of WaveNet was slow in generating data; however, the authors developed a much faster version later on in [10].

Recently, Generative Adversarial Networks (GANs) lead to a major breakthrough in many fields, including music generation. Li-Chia Yang [11] utilized GANs for melody generation but worked on MIDI sequences in the symbolic musical space. Hao-Wen Dong et al. [12] developed a GAN for multitack music generation. Yijie $\mathrm{Xu}$ et al. [13] developed a GAN melody generation system in which the generator is made by Bidirectional Long Short-Term Memory (Bi-LSTM) and the discriminator is made by LSTM. They utilize Bayesian for the optimization policy of their GAN. Yi Yu et al. [14] developed a lyrics-conditioned AI melody generator. Their system has three components: lyrics encoder network, melody generation network, and MIDI sequence tuner. The most important component is the melody generator GAN having a LSTM-based generator and discriminator. Their GAN is lyrics-conditioned so as to generate a satisfying melody that matches the input lyrics.

\subsection{Melody Timbre}

Melody "Timbre" is engaged with musical instruments sounds interpretation. The most common definition of timbre is that it is the quality or tone of sound and it distinguishes different voices. Daniel Mintz [15] developed a method for timbral synthesis based on timbral description rather than verbal description suggested earlier. Users of Daniel's system can specify their wanted timbre through standardized descriptors. Their timbral synthesis engine transform analysis equations into synthesis equations whose coefficients specify specific points in a timbral space.

Further working with timbral spaces, Allan Seago et al. [16] utilized weighted centroid localization (WCL) for proposing a timbre space search strategy expanding on their work in [17].

Timbre's has a huge role in the process of music. Carpentier et al. [18] introduced Orchidée, a timeefficient evolutionary orchestration algorithm. Marcelo Caetano et al. [19] developed an artificial immune system (AIS) called Opt-aiNet that aids in orchestration. Opt-aiNet is formulated as a multimodal system that searches for combinations of musical instrument sounds that minimize the distance to a reference sound encoded in a fitness function.

A very recent research of Koray Tahiroğlu et al. [20] presented a deformable musical instrument that performs real-time audio synthesis. Their instrument utilizes a deep learning model with GAN in generating audio samples. Their model has the ability to produce the realistic range of timbre of the trained data set in addition to the ability to generate new audio samples in real-time while providing novel characteristics of sounds. 


\subsection{Counterpoint Accompaniment}

Counterpoint accompaniment has a set of strict rules to generate more than one accompanying voices (typically two to four). Hence, rule-based algorithms were convenient for this type of accompaniment music. Gabriel Aguilera et al. [21] coded counterpoint rules using a computer algebra system by means of probabilistic logic.

Victor Padilla et al. [22] developed a system that imitates two-voice Palestrina-style counterpoint. They combined statistical generation and pattern discovery in the counterpoint generation process. Once patterns are organized according to a probabilistic distribution, two-voice counterpoint is generated into those patterns using a first-order Markov model. Cheng et al. [23] developed a system that imitates the nonlinear fashion of real composers in writing music rather than the chronological way adopted by past systems. They trained a convolutional neural network to generate partial musical scores, moreover, they introduced the use of blocked Gibbs sampling as an analogue to rewriting.

Very recently, Eric P. Nichols et al. [24] developed a contrapuntal music generation system based on a Neural Machine Translation (NMT) technique. Their system is concerned with Baroque counterpoint where they model the interaction between any two given voices as a translation process. In their technique, translating from one contrapuntal voice to another imposes some constraints on the latter, but doesn't define it completely.

\subsection{Chorale Accompaniment}

Chorale accompaniment is composed of four-part music lines; soprano and three other lower voices. F. Liang et al. [25] developed BachBot, which is a generative model based on deep LSTM for producing music in the style of Bach's chorales. Another Bach chorales imitative system is that of G. Hadjeres et al. [26] however, their system utilizes a dependency network accompanied with pseudo-Gibbs for the music sampling. T. Yamada et al. [27] compared between the use of Bayesian networks (BNs) and recurrent neural networks in chorale music generation highlighting the strengths and weaknesses of each.

Recently, Gilbert Wassermann and Mark Glickman [28] proposed an algorithm for harmonization in the style of Bach chorales. However, their algorithm takes the bass line as an input in contrast to the melody input that was adopted in earlier systems. They combine rule-based and machine learning techniques in their system. For learning harmonic structure, their system uses a hidden Markov model, and the system determines individual voice lines by optimizing a Boltzmann pseudolikelihood function. They utilize a weighted linear combination of constraint indicators to help them incorporate musical constraints in their Boltzmann function.

\subsection{Chord Accompaniment}

Chord accompaniment is a type of harmony that is most frequently used. This type of harmony is composed of a set of multiple harmonic notes that are played simultaneously. Liu et al. [29] utilized genetic algorithms (GA) in generating polyphonic accompaniment. In contrary to the human feedback fitness evaluation adopted earlier, they introduced a fitness function with several evaluation rules that are based on music theory. Later in [30], they combined data mining with GA. They introduced extracting chord patterns and styles of a specific composer by the help of data mining. They then fed the GA with the extracted patterns as genes. The fitness function of the used GA was also based on music theory. 
Navarro et al. [31], also embedded music theory in their chord generation system and combined it with an AIS. The penalty function of their AIS encodes musical rules to automatically generate chord progressions. Their AIS proposes multiple candidates for the next chord in a sequence that minimize the penalty function.

Brunner et al. [32] utilized two LSTM ANNs in polyphonic music generation; one for predicting chord progression based on a chord embedding, and another for generating polyphonic music based on the predicted chord progression. M.Nadeem [33] utilized two parallelly trained LSTM RNNs to generate musical notes along with their chords at the same time. By introducing this technique, they ensure that both, notes and chords, are closely related along all the training steps. Recently, Yu-Wei Wen and Chuan-Kang Ting [34] utilized genetic algorithms in developing a chord generator that follows the Bossa Nova style.

\subsection{Bass Accompaniment}

Bassline is closely related to chord accompaniment and in harmony with it. Liu et al. [29] system also utilizes GA to generate bass line in addition to chord. Their GA is run three times each with different fitness functions for each of the generated melody, chord and bass line. K. Komatasu et al. [35] system is another example of a bass-line generation model through genetic programming. BassNet [36] is a recently developed Bass Guitar tracks generator that utilize deep learning. This system is a conditional generator based on a given input musical pieces rather than generating from scratch. The system also provides the user with two-dimensional learned conditioning signal for mor control of the output bass line.

\subsection{Rhythm}

Rhythm represents the beats of the musical piece it determines the speed and style of the piece. By definition, rhythm is a regularly repeated pattern of musical beats, hence it is closely related to pattern mining. Liu et al. [30] developed an evolutionary composition system based on genetic algorithms and pattern mining. The extracted patterns then serve as the basis for chromosome representation. Hongru Liang et al. [37] developed a rhythm learning model based on ANNs.

\section{Discussion}

Table. 1 summarizes the frequently used CI techniques for automating each music composition task. We find that for melody pitch generation, DNN and GAN have the greatest share of recent applications of this task with the aim of approaching real compositions as much as possible. Most of the melody timbre applications depend on linear optimization due to the idea of timbral spaces. GAs are mostly suitable for the task of chord generation and its closely related bass line.

From the studied techniques we find that for the task of imitating or capturing a human composer's style or experience, CBR is a good candidate for the task. For predicting a new musical note in the melody given a previous one, then it is advised to use Markov chains. DNNs are optimal for keeping long-range temporal structure and temporal dependencies in a musical piece. The state-of-the-art GAN is used for developing nontraditional music composition applications such the generation of melody that confronts to given lyrics [14], or the creation of a deformable timbre synthesis instrument [20]. Counterpoint rules are better represented in a rule-based system although there are rather promising results from utilizing ANNs for this task, specially NMTs [24]. 
Table. 1: Summary of CI tecniques used in each music composition task

\begin{tabular}{|c|l|}
\hline Task & \multicolumn{1}{|c|}{ Techniques and Applications } \\
\hline Melody Pitch & $\begin{array}{l}\text { GA: 2016 [7] - CBR: 2017 [6] - DNN: 2016 [8], 2016 [9], 2017 [10] - Markov: 2017 [6] - GAN: 2017 } \\
{[11], 2018 \text { [12], 2020 [14], 2021 [13] }}\end{array}$ \\
\hline Melody Timbre & $\begin{array}{l}\text { GA: 2010 [18] - Linear Optimization: 2007 [15], 2010 [16], 2013 [17] - AIS: 2019 [19] - GAN: 2020 } \\
{[20]}\end{array}$ \\
\hline $\begin{array}{c}\text { Counterpoint } \\
\text { Accompaniment }\end{array}$ & ANN: 2019 [23], 2021 [24]- Rule-Based: 2010 [21] - Markov: 2018 [22] \\
\hline $\begin{array}{c}\text { Chorale } \\
\text { Accompaniment }\end{array}$ & ANN: 2017 [26], 2018 [27] - DNN: 2017 [25] - Rule-Based: 2020 [28] - \\
\hline $\begin{array}{c}\text { Chord } \\
\text { Accompaniment }\end{array}$ & GA: 2012 [29], 2015 [30], 2020 [34] - ANN: 2017 [32], 2019 [33] - AIS: 2015 [31] \\
\hline $\begin{array}{c}\text { Bass } \\
\text { Accompaniment }\end{array}$ & GA: 2012 [29], 2015 [35] - DNN: 2020 [36] \\
\hline Rhythm & GA: 2015 [30] - ANN: 2020 [37] \\
\hline
\end{tabular}

\section{Generated Music Evaluation}

Evaluating the music generated by machines is a nontrivial task. The most famous method of evaluation is the Turing test like method in which human listeners are introduced to several musical pieces; some are generated by computers and some were created by human composers. Human listeners should judge if a musical piece sounds realistic (created by human composer) or not.

Research in automatic evaluation of computer generated music is very recent [38]. Several quantitative metrics have been adopted in evaluating music generation systems. These metrics lie mainly within two categories; either model evaluators or music evaluators. The former is concerned with evaluating how successful is the model in processing data. Examples of model evaluation metrics include Loss, PPL (Perplexity), BLEU score, precision, recall, F1, etc. Music evaluation metrics require researchers to acquire music theory knowledge. Music metrics allow for judging the musicality of the generated musical pieces compared to original real pieces. Music metrics can be either pitch-related, rhythmrelated, chord/harmony-related, or style transfer-related.

Yang and Lerch [39] proposed a set of music metrics for assessing the musicality of machine generated music. Ji et al. [40] as well as Carnovalini and Rodà [41] provide extensive surveys on the topic of machine music evaluation, we refer the interested reader to their articles.

\section{Conclusion and Future Work}

Computer music composition is an interesting field of research that is full of challenges. In this paper we studied the problem of automating the various music composition tasks utilizing the recent computational intelligence techniques. We first introduced the classification of music composition tasks and their definitions. We then studied the several recent applications of CI in each music composition task. We also discussed the most suitable technique for performing music composition tasks. We finally mention the different methods for evaluating computer generated music.

There is a wide range of fruitful ideas for future research in the field of computer music composition. Primarily, more research can be conducted with GANs in the generation of accompaniment music and 
rhythm. Additionally, it would be interesting to complete on some of the systems studied in this paper that are concerned with automating one single musical task to turn them into full composers. For example, rhythm patterns learned in [37] can be embedded within the process of generating further complete music compositions.

In this survey we already highlighted the merge of different CI techniques together such as rule-based with GA and AIS, and such as CBR with Markov chains. However, the door is still open for research in combining more techniques together for achieving better quality for the generated compositions.

Another way of improvement of music composition applications is to make use of enhancements techniques proposed earlier in different research fields and apply it to the field of music composition. For example, the deep learning improvement technique proposed in [42] with the aim of reducing the error rate using auto encoder and GA can be applied in enhancing deep learning in music composition applications.

References

[1] A. V Joshi, Machine Learning and Artificial Intelligence. Cham: Springer International Publishing, 2020.

[2] A. E. Amin, "A Face Recognition System Based on Deep Learning (FRDLS) to Support the Entry and Supervision Procedures on Electronic Exams," International Journal of Intelligent Computing and Information Sciences, vol. 20, no. 1, pp. 59-75, 2020, doi: 10.21608/ijicis.2020.23149.1015.

[3] A. E. Amin, "A NOVEL IMAGE RETRIEVAL FRAMEWORK BASED ON KNOWLEDGE BASED SYSTEM," International Journal of Intelligent Computing and Information Sciences, vol. 14, no. 2, pp. 15-38, 2014, doi: 10.21608/ijicis.2014.15771.

[4] A. E. Amin, "A Medical Probabilistic Advisory System Based on Independent Artificial Intelligent Techniques to Support Decision-Making," International Journal of Intelligent Computing and Information Sciences, vol. 20, no. 1, pp. 28-43, 2020, doi: 10.21608/ijicis.2020.22091.1013.

[5] K. C. Ukaoha, O. Ademiluyi, J. Ndunagu, S. S. Daodu, and F. Osang, “Adaptive Neuro Fuzzy Inference System for Diagnosing Coronavirus Disease 2019 (COVID-19)," International Journal of Intelligent Computing and Information Sciences, vol. 20, no. 2, pp. 1-31, 2020, doi: 10.21608/ijicis.2020.40518.1027.

[6] M. Navarro-Cáceres, S. Rodríguez, D. Milla, B. Pérez-Lancho, and J. M. Corchado, “A user controlled system for the generation of melodies applying case based reasoning," Lecture Notes in Computer Science (including subseries Lecture Notes in Artificial Intelligence and Lecture Notes in Bioinformatics), Springer, vol. 10339 LNAI, pp. 242-256, 2017, doi: 10.1007/978-3319-61030-6_17.

[7] P. J. Ponce de León, J. M. Iñesta, J. Calvo-Zaragoza, and D. Rizo, "Data-based melody generation through multi-objective evolutionary computation," Journal of Mathematics and Music, Taylor \& Francis, vol. 10, no. 2, pp. 173-192, 2016, doi: 10.1080/17459737.2016.1188171.

[8] F. Colombo, S. P. Muscinelli, A. Seeholzer, J. Brea, and W. Gerstner, "Algorithmic Composition of Melodies with Deep Recurrent Neural Networks," 2016, doi: 10.13140/RG.2.1.2436.5683.

[9] A. van den Oord et al., "WaveNet: A Generative Model for Raw Audio," ArXiv, arXiv:1609.03499, 2016, [Online]. Available: http://arxiv.org/abs/1609.03499. 
[10] A. Van Den Oord et al., "Parallel WaveNet: Fast high-fidelity speech synthesis," in 35th International Conference on Machine Learning, ICML 2018, 2018, vol. 9, pp. 6270-6278.

[11] L.-C. Yang, S.-Y. Chou, and Y.-H. Yang, "MidiNet: a convolutional generative adversarial network for symbolic-domain music generation," in Proceedings of the 18th International Society for Music Information Retrieval Conference (ISMIR'2017), 2017, pp. 324-331.

[12] H.-W. Dong, W.-Y. Hsiao, L.-C. Yang, and Y.-H. Yang, "MuseGAN: multi-track sequential generative adversarial networks for symbolic music generation and accompaniment," in In Proceedings of the Thirty-Second AAAI Conference on Artificial Intelligence, 2018, pp. 34-41.

[13] Y. Xu, X. Yang, Y. Gan, W. Zhou, H. Cheng, and X. He, "A Music Generation Model Based on Generative Adversarial Networks with Bayesian Optimization," in Proceedings of 2020 Chinese Intelligent Systems Conference, 2021, pp. 155-164.

[14] Y. Yu, F. Harscoët, S. Canales, G. Reddy M, S. Tang, and J. Jiang, "Lyrics-Conditioned Neural Melody Generation," in MultiMedia Modeling, 2020, pp. 709-714.

[15] D. Mintz, Toward timbral synthesis: a new method for synthesizing sound based on timbre description schemes. Master's Thesis, University of California, Santa Barbara, 2007.

[16] A. Seago, S. Holland, and P. Mulholland, "A novel user interface for musical timbre design," in 128th Audio Engineering Society Convention 2010, May 2010, vol. 3, pp. 1816-1832.

[17] A. Seago, "A new interaction strategy for musical timbre design," in Music and HumanComputer Interaction, London: Springer London, 2013, pp. 153-169.

[18] G. Carpentier, G. Assayag, and E. Saint-James, "Solving the musical orchestration problem using multiobjective constrained optimization with a genetic local search approach," Journal of Heuristics, vol. 16, no. 5, pp. 681-714, Oct. 2010.

[19] M. Caetano, A. Zacharakis, I. Barbancho, and L. J. Tardón, "Leveraging diversity in computeraided musical orchestration with an artificial immune system for multi-modal optimization," Swarm and Evolutionary Computation, vol. 50, 2019.

[20] K. Tahiroğlu, M. Kastemaa, and O. Koli, “Al-terity: Non-Rigid Musical Instrument with Artificial Intelligence Applied to Real-Time Audio Synthesis," in Proceedings of the International Conference on New Interfaces for Musical Expression, Jul. 2020, pp. 337-342.

[21] G. Aguilera, J. L. Galán, R. Madrid, A. M. Martínez, Y. Padilla, and P. Rodríguez, "Automated generation of contrapuntal musical compositions using probabilistic logic in Derive," Mathematics and Computers in Simulation, vol. 80, no. 6, pp. 1200-1211, 2010.

[22] V. Padilla and D. Conklin, "Generation of two-voice imitative counterpoint from statistical models," International Journal of Interactive Multimedia and Artificial Intelligence (IJIMAI), vol. 5, pp. 22-32, 2018.

[23] C. Z. A. Huang, T. Cooijmans, A. Roberts, A. Courville, and D. Eck, "Counterpoint by convolution," Proceedings of the 18th International Society for Music Information Retrieval Conference, ISMIR 2017, pp. 211-218, 2017.

[24] E. P. Nichols, G. Micchi, S. Kalonaris, and A. Aljanaki, "Modeling baroque two-part counterpoint with neural machine translation," 2020.

[25] F. Liang, M. Gotham, M. Johnson, and J. Shotton, "Automatic stylistic composition of bach chorales with deep LSTM," in Proceedings of the 18th International Society for Music Information Retrieval Conference, ISMIR 2017, 2017, pp. 449-456.

[26] G. Hadjeres, F. Pachet, and F. Nielsen, "DeepBach: a steerable model for Bach chorales generation," in Proceedings of the 34th International Conference on Machine Learning - Volume 70, 2017, pp. 1362-1371.

[27] T. Yamada, T. Kitahara, H. Arie, and T. Ogata, "Four-part harmonization: comparison of a Bayesian network and a recurrent neural network," in Music Technology with Swing, 2018, pp. 
213-225.

[28] G. Wassermann and M. E. Glickman, "Automated harmonization of bass lines from Bach chorales: a hybrid approach," Comput. Music. J., vol. 43, no. 2-3, pp. 142-157, 2020.

[29] C.-H. Liu and C.-K. Ting, "Polyphonic accompaniment using genetic algorithm with music theory," 2012 IEEE Congress on Evolutionary Computation, pp. 1-7, 2012.

[30] C.-H. Liu and C.-K. Ting, "Music pattern mining for chromosome representation in evolutionary composition," 2015 IEEE Congress on Evolutionary Computation (CEC), pp. 2145-2152, 2015.

[31] M. Navarro-Cáceres, M. Caetano, G. Bernardes, L. N. de Castro, and J. M. Corchado, "Automatic generation of chord progressions with an artificial immune system," in Evolutionary and Biologically Inspired Music, Sound, Art and Design, 2015, pp. 175-186.

[32] G. Brunner, Y. Wang, R. Wattenhofer, and J. Wiesendanger, "JamBot: music theory aware chord based generation of polyphonic music with LSTMs," in 2017 IEEE 29th International Conference on Tools with Artificial Intelligence (ICTAI), Nov. 2017, pp. 519-526.

[33] M. Nadeem, A. Tagle, and S. Sitsabesan, "Let's make some music," in 2019 International Conference on Electronics, Information, and Communication (ICEIC), Jan. 2019, pp. 1-4.

[34] Y.-W. Wen and C.-K. Ting, "Composing Bossa Nova by Evolutionary Computation," in 2020 International Joint Conference on Neural Networks (IJCNN), Jul. 2020, pp. 1-8, doi: 10.1109/IJCNN48605.2020.9207713.

[35] K. Kunimatsu, Y. Ishikawa, M. Takata, and K. Joe, "A music composition model with genetic programming -a case study of chord progression and bassline," in International Conference on Parallel and Distributed Processing Techniques and Applications, PDPTA'15, Jul. 2015, pp. 256-262.

[36] M. Grachten, S. Lattner, and E. Deruty, "BassNet: A Variational Gated Autoencoder for Conditional Generation of Bass Guitar Tracks with Learned Interactive Control," Applied Sciences, vol. 10, no. 18, 2020, doi: 10.3390/app10186627.

[37] H. Liang, W. Lei, P. Y. Chan, Z. Yang, M. Sun, and T.-S. Chua, "PiRhDy: Learning Pitch-, Rhythm-, and Dynamics-Aware Embeddings for Symbolic Music," in Proceedings of the 28th ACM International Conference on Multimedia, New York, NY, USA: Association for Computing Machinery, 2020, pp. 574-582.

[38] A. Eigenfeldt, A. Burnett, and P. Pasquier, "Evaluating Musical Metacreation in a Live Performance Context," in Proceedings of the Third International Conference on Computational Creativity, 2012, pp. 140-144.

[39] L.-C. Yang and A. Lerch, "On the evaluation of generative models in music," NEURAL COMPUTING \& APPLICATIONS, vol. 32, no. 9, SI, pp. 4773-4784, May 2020, doi: 10.1007/s00521-018-3849-7.

[40] S. Ji, J. Luo, and X. Yang, "A Comprehensive Survey on Deep Music Generation: Multi-level Representations, Algorithms, Evaluations, and Future Directions.” 2020.

[41] F. Carnovalini and A. Rodà, "Computational Creativity and Music Generation Systems: An Introduction to the State of the Art," Frontiers in Artificial Intelligence, vol. 3, p. 14, 2020, doi: 10.3389/frai.2020.00014.

[42] F. Habeeb, S. Abuelenin, and S. Elmougy, "REDUCING ERROR RATE OF DEEP LEARNING USING AUTO ENCODER AND GENETIC ALGORITHMS," International Journal of Intelligent Computing and Information Sciences, vol. 16, no. 4, pp. 41-53, 2016, doi: 10.21608/ijicis.2016.19823. 